\title{
Discours
}

Revue de linguistique, psycholinguistique et

informatique. A journal of linguistics, psycholinguistics and computational linguistics

26 | 2020

Varia

\section{Modèles d'organisation thématique des paragraphes et entre les paragraphes, à l'épreuve de la Rectorique de Cyceron}

\section{Guy Achard-Bayle et Ondřej Pešek}

\section{OpenEdition}

\section{Journals}

\section{Édition électronique}

URL : https://journals.openedition.org/discours/10794

DOI : $10.4000 /$ discours. 10794

ISSN : 1963-1723

\section{Éditeur :}

Laboratoire LATTICE, Presses universitaires de Caen

\section{Référence électronique}

Guy Achard-Bayle et Ondřej Pešek, « Modèles d'organisation thématique des paragraphes et entre les paragraphes, à l'épreuve de la Rectorique de Cyceron », Discours [En ligne], 26 | 2020, mis en ligne le 02 novembre 2020, consulté le 03 février 2023. URL : http://journals.openedition.org/discours/10794 ; DOI : https://doi.org/10.4000/discours.10794

\section{(c) (i) $\odot$}

Creative Commons - Attribution - Pas d'Utilisation Commerciale - Pas de Modification 4.0 International - CC BY-NC-ND 4.0

https://creativecommons.org/licenses/by-nc-nd/4.0/ 

Revue de linguistique, psycholinguistique et informatique

\section{Modèles d'organisation thématique des paragraphes et entre les paragraphes, à l'épreuve de la Rectorique de Cyceron}

Guy Achard-Bayle

CREM (Centre de recherche sur les médiations)

Université de Lorraine

Ondřej Pešek

Université de Bohême du Sud

Guy Achard-Bayle, Ondřej Pešek, «Modèles d'organisation thématique des paragraphes et entre les paragraphes, à l'épreuve de la Rectorique de Cyceron», Discours [En ligne], 26 | 2020, mis en ligne le 2 novembre 2020.

URL: http://journals.openedition.org/discours/10794

Titre du numéro: Varia

Coordination: Nicolas Hernandez \& Lydia-Mai Ho-Dac

Date de réception de l'article: 05/12/2019

Date d'acceptation de l'article: 02/04/2020 



\title{
Modèles d'organisation thématique des paragraphes et entre les paragraphes, à l'épreuve de la Rectorique de Cyceron
}

\author{
Guy Achard-Bayle \\ CREM (Centre de recherche sur les médiations) \\ Université de Lorraine \\ Ondřej Pešek \\ Université de Bohême du Sud
}

Cet article porte sur la question de l'organisation thématique des paragraphes et entre les paragraphes, et comporte deux parties: dans une première partie, nous abordons cette question à travers deux modèles théoriques français et tchèques. Dans la seconde partie de l'article, ces modèles sont mis à l'épreuve du corpus, par une analyse textuelle. Cette analyse sera menée dans une double perspective: la première, historique, consistera à étudier les marques de segmentation telles qu'elles ont été utilisées dans un manuscrit médiéval, et ont joué le rôle de «précurseurs» de marqueurs du paragraphe; la seconde, contemporaine, portera sur l'édition moderne du même texte. En projetant la structure thématique et compositionnelle sur l'articulation du texte en paragraphes, nous chercherons à illustrer le rôle de ces segments dans le dispositif organisateur du texte.

Mots clés: paragraphe, progressions thématiques, structure compositionnelle, manuscrit médiéval

The article addresses the issue of the topical organisation of the paragraph and between paragraphs. It is divided into two parts: in the first part, we approach the issue through two theoretical models, one French and the other Czech. In the second part of the article, the models are tested on a corpus study. The study applies a two-pronged approach: the first, historical, consists in analysing the marks of segmentation as they were used in a medieval manuscript, playing the role of "precursors" of markers of the paragraph; the second, contemporary, focuses on the modern edition of the same text. By projecting the thematic and compositional structure on the division of the text into paragraphs, we illustrate the role these segments play as text-organising devices.

Keywords: paragraph, thematic progressions, compositional structure, medieval manuscript

\section{Introduction}

Notre article portera sur la question de l'organisation thématique du paragraphe, autrement dit la question de la continuité ou de la rupture thématique dans les paragraphes et entre les paragraphes; il comportera deux parties: une première de cadrage ou de problématisation, une seconde d'illustration et d'analyses textuelles.

Dans la première partie, nous aborderons la question posée, essentiellement, à travers deux modèles théoriques, français et tchèque: celui d'Adam (2017) et celui 
de Daneš (suivant notamment Daneš, 1994), sachant néanmoins, d'une part, que ces modèles se sont nourris d'autres recherches internationales, issues notamment des travaux de Longacre (par ex. Longacre, 1979) ou encore de Van Dijk (par ex. Van Dijk, 1973); d'autre part, que d'autres travaux pourront inspirer nos analyses, notamment ceux issus du Modèle d'architecture textuelle (MAT), tel qu'il a été conçu et illustré par Luc et Virbel (2001) ou encore Jacques (2005), dont nous partageons l'idée que le dispositif de marquage typographique, qui participe de la matérialité textuelle, ne se réduit pas à une fonction esthétique ${ }^{1}$.

Pour nos deux modèles, français et tchèque, nous dirons, suivant leur dimension épistémologique, qu'ils sont soit issus du côté tchèque, soit inspirés du côté français, de l'École de Prague de la seconde génération (voir Pešek, 2010), celle de la Perspective fonctionnelle de la phrase, et plus particulièrement des travaux de Daneš, qui ont fait émerger et ont permis de développer la question des progressions thématiques (voir Daneš, 1974; voir également Firbas, 1964 et 1992).

Ces cadres historiques et épistémologiques posés, nous pouvons préciser que notre première partie sera consacrée, en deux sections, à présenter et, dans la mesure du possible, comparer ces modèles français et tchèques du point de vue des opérations ou des opérateurs de composition ou de structuration textuelle; nous verrons que le modèle hérité de Daneš sur le rôle du paragraphe dans les progressions thématiques pose divers problèmes, qui ne font, aujourd'hui encore, qu'émerger.

Pour aller de l'avant, dans notre seconde partie, ces modèles, et particulièrement le pragois, seront mis à l'épreuve du corpus, par une analyse textuelle. Cette analyse sera menée dans une double perspective: la première, historique, consistera à étudier les marques de segmentation telles qu'elles ont été utilisées dans un manuscrit médiéval, et ont joué le rôle de "précurseurs» de marqueurs du paragraphe; la seconde, contemporaine, portera sur l'édition moderne du même texte. En projetant la structure thématique et compositionnelle sur l'articulation du texte en paragraphes, nous chercherons à illustrer le rôle de ces segments dans le dispositif organisateur du texte.

\section{De quelques théories françaises et tchèques du paragraphe}

Comme annoncé, nous présentons, dans cette première partie, diverses recherches françaises et tchèques qui ont porté sur le paragraphe. Chacune des deux sections mettra en avant, sans exclusive, une étude particulière: la première Adam (2018), la seconde Daneš $(1994)^{2}$.

1. Jacques (2005: en ligne, section 2.2 , § 17-25) défend ainsi «l'idée que tout ce qui signifié par la forme matérielle du texte possède une relation d'équivalence avec une formulation discursive», autrement dit une «métaphrase» où «les référents dont elle parle sont dans le texte même où elle apparaît et non des segments du monde dont parle le texte». Luc et Virbel (2001) parlent ainsi, de leur côté, d'objet textuel.

2. Les auteurs remercient Klára Žemličková (doctorante en linguistique textuelle) pour la traduction de cet article. 


\subsection{Le paragraphe, «mal-aimé» des linguistes français - entre autres}

Il résulte de la lecture d'Adam (2018), et des nombreux auteurs qu'il cite, que le paragraphe n'est pas un objet ignoré des linguistes; il est au contraire bien présent dans les travaux que nous citerons par la suite, qui couvrent plusieurs décennies... Mais, sinon méconnu, le paragraphe est mal connu, dans la mesure où, s'il se présente comme une «évidence», il est depuis longtemps et reste toujours "un objet flou», comme le dit Adam au tout début de son ouvrage (Adam, 2018: 9).

C'est un leitmotiv des linguistes, dont Adam (2018) se fait l'écho: de Harris, au début des années 1950 (voir Harris, 1952), à, tout récemment, Algee-Hewitt et al. (2015), en passant, dans les années 1980, par Mitterand (1985) et Laufer (1985); ou encore Bessonnat qui note en 1988 qu' «un constat s'impose: le paragraphe semble être une donnée linguistique admise par tous et rarement interrogée en elle-même» (Bessonnat, 1988: 81); la question, ou la tâche qui nous reste, est donc bien d'interroger le paragraphe «en lui-même».

Le constat est donc partagé par de nombreux auteurs, et, en ce qui concerne ceux que nous venons de citer, il s'étale sur quelque soixante-dix ans. Or, ce constat fait, reste ce paradoxe: certains de ces auteurs ou d'autres chercheurs encore ont pu consacrer plusieurs travaux, parfois même toute une phase de leur activité, à cet objet linguistique mal connu, comme Arabyan dont les études sur la question s'étalent de 1994 à 2016.

Mais il y a un autre paradoxe que relève Adam (2018: 9), et qui concerne maintenant tout particulièrement les linguistes qui se réclament du cadre historique et épistémologique que nous avons posé en introduction, les linguistes du texte; l'autre constat d'Adam, qui révèle ce second paradoxe, est en effet le suivant: «Le peu d'intérêt des linguistes pour le paragraphe est d'autant plus étonnant qu'il s'agit d'un poste d'observation idéal des liages interphrastiques et du passage de la phrase au texte»; ce constat fait écho à celui d'Algee-Hewitt et al. (2015): «les paragraphes demeurent une échelle ${ }^{3}$ insuffisamment étudiée» (cité par Adam, 2018: 9).

Adam remarque encore que si le paragraphe est, si l'on peut dire, «mal aimé» des linguistes, il ne l'est pas ou ne l'a pas été des rhétoriciens ou encore des psychologues cognitivistes (comme Schank, 1974); pour les linguistes, à l'inverse, il est resté ou reste un objet flou malgré sa visibilité graphique, autrement dit sa vi-lisibilité: il «pécherait» donc par excès de forme, autrement dit d'apparence, et manque de «fond $» .$.

Ce qui obligerait les linguistes à lui trouver un «fond» (ou un «fonds») de propriétés: ainsi, au plan strictement linguistique, c'est-à-dire des points de vue syntactico-sémantique et sémantico-discursif, on peut commencer par dire qu'il est partagé entre deux niveaux d'organisation: «une longue phrase et un court

3. Il s'agit d'un niveau médian (ou «méso» comme le dit Adam pour sa part) dans l'échelle des niveaux de la structuration textuelle. 
discours ${ }^{4}$; mais ces deux niveaux ne sont pas continus, ou intégrants au sens de Benveniste (1966): pour ce dernier, en effet, l'intégration joue entre niveaux de composition des unités linguistiques, les unités propres à un niveau (phonème, morphème, syntagme) devenant des composants du niveau supérieur. Or, note Adam (2018: 10) : «une telle dilution dans une unité inférieure (grande phrase) ou supérieure (petit discours) ne permet pas de définir le paragraphe et de l'étudier comme une unité intermédiaire spécifique de composition des textes écrits».

De ce fait, Adam se propose, pour ainsi dire, de «franchir la limite» entre les différents niveaux d'organisation que sont la phrase et le texte, si l'on suit le fameux chapitre de Benveniste sur «Les niveaux de l'analyse linguistique» (Benveniste, 1966: chap. X) ${ }^{5}$. Mais, dans l'esprit de Benveniste, on pourrait encore citer, comme le fait Adam (2018: 18), Culioli (1984: 10):

Le texte écrit nous force, de façon exemplaire, à comprendre que l'on ne peut pas passer de la phrase [...] à l'énoncé, par une procédure d'extension [...] le paragraphe n'est pas une variété d'énoncé transphrastique; l'énoncé (ou le paragraphe) n'est pas une unité plus haute $[. .$.$] à laquelle on accéderait comme on gravit une échelle.$

Le propos d'Adam (2018) est donc de poursuivre son projet en faveur d'une «linguistique transphrastique ${ }^{6}$, sachant (i) que celle-ci se situe à trois niveaux: micro-, méso- et macrotextuel, et (ii) que le paragraphe tient le rang moyen (méso), qui reste donc à instruire.

Dans le détail, le projet d'Adam se développe comme suit: il propose tout d'abord une «théorie» autrement dit une «linguistique textuelle du paragraphe», puis il en vérifie (longuement et largement) la validité sur ou par des exemples... C'est ainsi qu'Adam situe - et donc définit théoriquement - le paragraphe au même niveau méso que les macro-propositions et les séquences, sachant toutefois qu'il se trouve bien entre elles: d'une part «il est toujours constitué d'au moins une macro-proposition»; d'autre part, si une séquence est une suite ordonnée ${ }^{7}$ de macro-propositions, alors le paragraphe, qui souvent donc représente une macro-proposition, est constitutif (au sens benvenistien) de la séquence.

La difficulté qui reste toutefois, est que si les macro-propositions et les séquences sont des unités ou des modes d'organisation méso, elles le sont en tant qu'opérations

4. Adam (2018) cite et traduit ici Longacre (1979: 116).

5. Pour Benveniste (1966: 128-130), ces deux niveaux ne sont pas continus en termes de composition et d'intégration, suivant les principes - ou les opérations - dont nous avons parlé plus haut: «C'est là le dernier niveau que notre analyse atteigne, celui de la phrase [...]. Avec la phrase une limite est franchie, nous entrons dans un nouveau domaine. [...] La phrase appartient bien au discours. C'est même par là qu'on peut la définir : la phrase est l'unité du discours...». Legallois (2006) propose une lecture alternative à ladite «limite» de Benveniste.

6. Inspiré dans nombre de ses ouvrages de Benveniste; voir la citation ci-dessus, que nous complétons ici, sachant qu'alors, la «limite» franchie, «la phrase est l'unité du discours [...]. La phrase est une unité, en ce qu'elle est un segment du discours...» (Benveniste, 1966: 130).

7. Typée dans Adam (2017). 
ou opérateurs sémantiques, alors que le paragraphe reste une unité compositionnelle graphique, un segment, quand les deux autres, définies comme suites de propositions, le sont, pour le coup, par leur contenu propositionnel ${ }^{8}$.

Ceci ne veut pourtant pas dire que le paragraphe n'intervient pas à ce niveau, sémantico-discursif, ou pragmatique, bien au contraire. Mais une fois encore, on voit ici toute la complexité du champ et de l'objet d'analyse, d'où la difficulté du projet que se fixe Adam (2018). Tant il est vrai que la vi-lisibilité, autrement dit l'évidence graphique du paragraphe, est bien une image de l'organisation du contenu du texte - et de ses contenus ${ }^{9}$; ainsi, pour Adam (2018: 15):

Le paragraphe est la trace graphique de cette opération transphrastique de regroupements ${ }^{10}$ de propositions et de phrases en vue de constituer des unités significatives de second niveau (si l'on considère la phrase comme un premier niveau de structuration selon des patrons graphiques et morphosyntaxiques propres à chaque langue).

Il est temps maintenant de dire et de voir que l'organisation thématique joue un rôle crucial dans ces regroupements ${ }^{11}$. Pour rester avec Adam, on remarquera que celui-ci, dans un ouvrage quelque peu antérieur à celui qu'il consacre exclusivement au paragraphe, note à ce propos que si, considéré du point de vue «de sa macrostructure sémantique» ${ }^{12}$, «un texte peut plus ou moins toujours être résumé par un titre», alors «des portions de texte sont également résumables sous forme de blocs sémantiques thématiques [afin de] rendre vi-lisibles les unités du plan du texte» (Adam, 2011: 226); ce qui est, très explicitement, la position de Bessonnat (1988: 85, section «Programmer»; nous soulignons):

Le découpage en paragraphes est le moyen de contrebattre l'organisation linéaire du texte ${ }^{13}$. Le phénomène est très sensible, si on prend en considération la structure thématique [dérivée, constante...]. Aller à la ligne est une instruction donnée au destinataire que l'on pourrait expliciter de la façon suivante: «Si vous continuez à traiter le présent discours, commencez quelque chose d'autre».

Si la question des progressions thématiques est ainsi ouvertement abordée dans la citation précédente, on ne trouve pour autant dans l'étude complète de Bessonat aucune référence à Daneš. C'est donc un peu et paradoxalement par le constat d'une

8. Voir dans Adam (2017: 53) le schéma du «palier méso-textuel».

9. Pour le passage du singulier au pluriel de contenu, voir Legallois (2006a) sur unité et unités du texte.

10. Blocs de sens constitués au niveau de la mémoire immédiate chez les psychologues: voir les chunks de Miller (1956)

11. L'organisation thématique, mais pas seulement: voir dans Adam (2017), le rôle joué de leur côté par la prise en charge énonciative, l'orientation argumentative, ou encore l'ordonnancement séquentiel, pour expliquer l'organisation, la fragmentation en paragraphes, donc le changement de paragraphe.

12. Qui est le topic $d u$ discours chez Eco (1985 [1979]).

13. Voir aussi l'organisation «réticulaire» du texte chez Legallois (2006b). 
absence que nous faisons ici une transition vers la section tchèque de notre première partie. D'autant que, si Daneš est cité (une fois) dans Adam (2011) ou (2018) pour ses progressions thématiques, ce n'est pas spécialement ou explicitement en rapport avec la question du paragraphe; or Daneš n'est pas davantage cité par Adam (2017) alors même que le rôle du thème dans la constitution du paragraphe est souligné dans cet ouvrage (Adam, 2017: 53-54) ${ }^{14}$.

\subsection{Daneš, le paragraphe et la linguistique pragoise ${ }^{15}$}

Pour Daneš, le paragraphe est plus qu'une «unité graphique», et même qu'une unité sémantique; dans une orientation - qu'on pourrait dire, déjà - cognitiviste, le paragraphe est pour lui, plus qu'une opération additionnelle [une opération «de plus» dans l'article en tchèque], «un des principes créateurs du texte [qui opère] déjà au niveau préverbal dans la mise en place du plan textuel» (Daneš, 1994: 1; nous soulignons).

En amont, Daneš rappelle que selon lui (Daneš, 1989), interviennent cinq niveaux de structuration sémantique du texte: ceux des relations (i) sémantiques propositionnelles, (ii) isotopiques et (iii) thématiques ${ }^{16}$, auxquels s'ajoutent le niveau (iv) des relations compositionnelles, et enfin, celui (v) des relations (pragmatiques) d'interaction.

Du point de vue qui nous intéresse ici (c'est-à-dire au troisième niveau), chaque paragraphe a son thème, et souvent ce thème est en relation avec l'hyperthème qui constitue l'unité thématique du texte: soit chez Adam le «thème-titre», qu'il soit explicite ou non; chez Daneš, il s'agit de «l'hyperthème absolu», car le paragraphe peut aussi être «hyperthématique» en ce qu'il se décline ensuite, dans le paragraphe ${ }^{17}$, en sous-thèmes ou thèmes dérivés: ceci vaut notamment pour le texte descriptif, explicatif ou instructionnel.

Il reste que Daneš ne s'attarde pas sur les relations thématiques entre paragraphes; il note bien que son travail est précurseur en la matière, donc exploratoire, et se consacre ainsi davantage aux progressions thématiques internes au paragraphe, se contentant de proposer dans la seconde partie de son article une «démonstration illustrée» de ce qui détermine la composition et la progression thématiques dans (plus qu'entre) les paragraphes: pour ce faire, Daneš s'inspire, notamment, de Van Dijk (1973) et surtout de Lindeberg (1991), laquelle assigne aux énoncés diverses fonctions qui justifient leur succession, leur enchainement:

14. Adam cite Arabyan (2003) et Gardes-Tamine et Pellizza (1998) sur cette question des paragraphes thématiques.

15. Comme nous l'avons annoncé, nous nous référons, sauf mention contraire, à Daneš (1994). La traduction française de cet article a été réalisée par Klára Žemličková (voir note 2).

16. Autrement dit Aktuální členění větné, chez Mathesius (1969 [1929]), Functional Sentence Perspective chez Firbas (1992) - en français: Perspective fonctionnelle de la phrase (PFP).

17. Et entre les paragraphes, nous y revenons plus bas. 
Explanation, explication, specification; preparation, presupposition, precondition; contrast, confirmation, illustration, comparation [Van Dijk], assert, assert-describe, cause, compare, conclude, contrast, evaluate, generalize, metastatement, qualify, restate, result, specify (analyze, define, exemplify, enumerate), suggest, summarize.

(Lindeberg, 1991, in Daneš, 1994: 11; en anglais dans l'original)

Ainsi, la question de la PFP est mise en relation avec ce que Daneš appelle, d'après Van Dijk et Lindeberg, les relations ou les fonctions «rhétoriques»; sachant toutefois que pour lui, qui y consacre la dernière partie de son article, ces relations entre paragraphes relèvent du niveau compositionnel (mentionné supra).

Pour Daneš donc, les deux niveaux, thématique et compositionnel, interagissent. Notre analyse d'un extrait de Cicéron (infra) montrera que le mode compositionnel (série énumérative) met nécessairement en jeu les relations hypo-thématiques.

Cependant, ce point de vue «rhétorique» laisse en suspens le problème de la pertinence de la notion d'hyperthème, sachant que par définition, la relation hyperthématique est constitutive même de la textualité ou de la textualisation, donc du produit texte - voire réductible à elle et à lui... Voyons alors, par défaut, la problématique telle qu'elle est développée par Daneš (1994), et notamment la question des progressions thématiques internes aux paragraphes.

Daneš commence par noter que la division d'un texte en paragraphes n'est pas strictement contrainte, autrement dit réductible à (ou repérable par) un facteur formalisable ${ }^{18}$; il est toutefois possible d'identifier des endroits où la division est obligatoire, ou alors interdite - mais Daneš ne donne d'exemples pour aucun des deux cas, ce qui nécessite de notre part une «mise à l'épreuve», que nous tenterons de mettre en ouvre dans notre seconde partie.

Ceci dit, Daneš insiste sur le rôle du paragraphe dans l'organisation thématique générale, et sur l'importance de sa fonction interne de sur- ou sous-thématisation; ainsi, le paragraphe est un opérateur de cohérence, et c'est sa cohérence interne qui en définit les limites: de la sorte, se fait le lien du graphique au sémantique.

Pour finir, nous dirons que le grand mérite de l'article de Daneš, son caractère inédit, est qu'il propose toute une typologie de progressions thématiques, ou mieux de paragraphes suivant leur progression thématique, c'est-à-dire «selon les relations entre le thème du paragraphe (O-thème ou hyperthème) et les thèmes des énoncés (V-thème)» (Daneš, 1994: 8) ${ }^{19}$.

Voici le détail de cette typologie:

18. Ce qui n'est pas sans rappeler (voir supra) la problématique du passage de la phrase au texte chez Benveniste (1966).

19. O-thème: thème de paragraphe (odstavcové téma); $V$-thème: thème d'énoncé (výpovědní téma). 
- Type I: Le paragraphe avec $\mathrm{O}$-thème stable; les $\mathrm{V}$-thèmes présentent une certaine récurrence de l'O-thème; cette organisation est basée sur la progression à thème constant (voir la thematic iteration chez Enkvist, 1974).

- Type II: Les paragraphes à O-thème dérivé, pour lesquels Daneš propose deux sous-types: * Sous-type 1: les énoncés thématisent (par leur V-thème) des aspects différents de l'O-thème.

* Sous-type 2: l'O-thème est éclaté en thèmes partiels, O-thème 1 et $\mathrm{O}$-thème 2, etc., qui sont traités un par un dans les $\mathrm{V}$-thèmes partiels; cela signifie qu'on fait et donc qu'on voit évoluer tout d'abord $\mathrm{O}$-thème 1 (dans la chaîne des énoncés à $\mathrm{V}$-thèmes), ensuite $\mathrm{O}$-thème 2.

Du point de vue «stylistique» (celui des typologies textuelles et séquentielles d'Adam, 2017), les types I et II apparaissent surtout dans les textes narratifs et descriptifs (voir encore Červenka, 1983 et Enkvist, 1974).

- Type III: les paragraphes «cadres»; l'O-thème joue ici le rôle d'un cadre (de contenu) au sens où il contient un ensemble d'éléments (objets, états, activités, événements, etc.) qui s'exposent graduellement ou évoluent en des thèmes partiels. Il s'agit dans ce cas de progression thématique linéaire (la cohérence thématique suit la progression rhématique), autrement dit de la thématisation en série du rhème précédent (voir la notion de «cohérence syntagmatique» chez Červenka, 1983).

- Type IV: il s'agit des paragraphes où l'O-thème 1 se transforme en un autre O-thème 2 ; le rhème d'un énoncé 1 se thématise et s'expose ainsi comme un nouvel O-thème 2; ensuite il se développe (ou se particularise); mais cela n'est possible qu'à la condition que le contenu du rhème thématisé (O-thème 2 ) dépasse le cadre de l'O-thème 1 ; sinon il ne s'agit que la particularisation de l'O-thème 1 .

À la fin de son article, Daneš aborde la question de la succession des paragraphes, ou plus exactement de la division en paragraphes. L'analyse est relativement succincte et donc quelque peu approximative, mais elle permet de réinvestir la question de l'hyperthème. En effet, Daneš distingue trois types de continuité ou de progression entre les paragraphes (progression thématique dérivée, éclatée ou linéaire), mais dans tous les cas la continuité entre paragraphes se justifie par une relation hyperthématique.

Aussi peut-on conclure, sur la base des études présentées et analysées ici, que le paragraphe fait partie du dispositif des moyens formels qui organisent le texte en vue de guider le traitement de l'information. Du fait même du marquage typographique, le paragraphe devient une unité pertinente de la structure thématique et compositionnelle du texte ${ }^{20}$. C'est ainsi que se voient corrélées la typographie et la sémantique textuelle: les éléments typographiques créent des unités ${ }^{21}$ qui représentent des éléments constitutifs des plans de texte (dans le sens d'Adam, passim). En même temps, le marquage typographique au niveau du texte n'est

20. Il s'agit des niveaux iii et iv de structuration sémantique textuelle de Daneš, présentés supra.

21. Autrement dit: sans point et majuscule, il n'y a pas de phrase. 
jamais gratuit: bien que la délimitation ne soit pas gouvernée par une règle forte et contraignante (à l'instar des règles syntaxiques), elle ne peut en principe concerner que des segments structurellement pertinents.

Ainsi, tous les marqueurs de la cohésion textuelle, qu'ils soient lexicaux, grammaticaux ou typographiques, fonctionnent en synergie et se complètent mutuellement.

\section{3. Étude de cas - structures thématiques et compositionnelles de la Rectorique de Cyceron de Jean d'Antioche}

L'étude de cas que nous présentons ici a pour objectif de mettre les éléments théoriques formulés ci-dessus à l'épreuve du texte concret. Le texte que nous analyserons est un extrait du premier livre de la Rectorique de Cyceron. Il s'agit d'une traduction en ancien français du traité cicéronien De Inventione effectuée par Jean d'Antioche en 1282. Le choix du texte est motivé par trois raisons principales. D'abord, le texte cicéronien présente une structuration particulière, à dominante sérielle. Les séries énumératives pouvant atteindre un degré d'enchâssement profond, l'analyse du texte permet de faire ressortir clairement les rapports entre l'organisation thématique et le marquage typographique. La deuxième raison est de nature philologique. Nombreuses sont en effet les études qui abordent le manuscrit ancien en tant qu'objet «paléographique», tout en accentuant la dimension artistique de la typographie ancienne. En revanche, il existe à notre connaissance peu de travaux qui se proposent d'analyser les manuscrits anciens en appliquant le cadre théorique et conceptuel de la linguistique contemporaine. Notre étude, qui s'inscrit dans la lignée de la linguistique textuelle diachronique (Combettes, 2015) vise à combler, ne serait-ce que très partiellement, cette lacune. La troisième raison se situe au niveau méthodologique. Outre la version manuscrite, nous analyserons également l'édition moderne du texte: nous comparerons l'usage des moyens typographiques du marquage textuel utilisés dans le manuscrit original avec ceux qu'emploie l'édition moderne établie par E. Guadagnini. La mise en parallèle du manuscrit et de l'édition moderne permet ainsi de mieux circonscrire les éléments textuels pertinents pour l'analyse. Cette approche comparative est donc apte à tester l'hypothèse selon laquelle les segments formels (qu'il s'agisse du paragraphe dans l'édition moderne ou d'un paquet de propositions marqué autrement que par l'alinéa dans le manuscrit) sont délimités en fonction du rôle qu'ils jouent au niveau du plan du texte (Adam, 2018: 55-105).

La méthode d'analyse procède des principes que nous venons de rappeler. D’abord, nous décrirons l'extrait du point de vue de sa structuration compositionnelle et thématique. Ensuite, nous présenterons les moyens organisateurs du texte en nous focalisant plus particulièrement sur les éléments typographiques (paragraphes et autres). La troisième phase de notre analyse consistera en une synthèse des deux premières: le fonctionnement de ces unités typographiques sera corrélé avec la structure thématique et compositionnelle relevée. 


\subsection{Le texte analysé - informations historiques et éditoriales}

Le traité rhétorique de Cicéron De Inventione ${ }^{22}$ a été traduit en 1282 par Jean d'Antioche. Conformément à la tradition médiévale, le texte fait partie d'un ensemble appelé $L a$ rectorique de Marc Tulles Cyceron dans lequel figure également la traduction de la Rhetorica ad Herennium, ouvrage faussement attribué à Cicéron et jugé complémentaire du De Inventione. Selon Delisle (1906), la traduction n'a été conservée que dans un seul manuscrit qui parait dater de la fin du XIII siècle et qui se trouve au Musée Condé ( $n^{\circ}$ 433) au Château de Chantilly. Nous disposons de deux éditions modernes de ce manuscrit, l'une préparée par Van Hoecke, disponible uniquement dans une version électronique publiée dans le cadre du corpus BFM (Base de français médiéval), l'autre préparée par Guadagnini et publiée en 2009 sous forme de livre imprimé. Comme nous l'avons précisé ci-dessus, c'est cette dernière que nous allons utiliser dans cette étude.

\subsection{Principes compositionnels - configuration sérielle}

L'extrait que nous analyserons ici (I, 10-16 ${ }^{23}$ ) est un exposé relatif à la question des «états de la cause». «État de la cause» est un terme spécialisé de la rhétorique. Il désigne le type de question d'où naît la cause et qui détermine fondamentalement la nature du discours. Cicéron dans De Inventione utilise le terme constitutio que Jean d'Antioche traduit par constitucion ${ }^{24}$. Le terme «état de la cause» est celui de Achard qui l'utilise dans sa traduction française moderne du texte cicéronien. L'extrait (I, 10-16) est représentatif de la configuration prototypique du texte cicéronien: l'exposé de la matière est organisé sous forme de séries énumératives. La notion «état de la cause» représente le thème-titre ${ }^{25}$ de l'exposé, le thème-titre est progressivement divisé en sous-thèmes dont la présentation est organisée selon le mode sériel.

La série énumérative n'est pas une séquence strictement typée ${ }^{26}$. Il s'agit plutôt d'une configuration libre qui émerge ${ }^{27}$ du texte sur la base de plusieurs indices de natures diverses. En dépit de la diversité structurelle des séries énumératives effectivement attestées, on peut identifier deux éléments de base dont l'actualisation dans le texte permet de déclencher l'émergence d'une série énumérative:

22. De Inventione est le premier traité rhétorique de Cicéron, écrit dans sa jeunesse vers 83 av. J.-C., et constitue l'un des premiers ouvrages consacrés à cet art rédigés en latin. Cicéron y fait la synthèse des méthodes rhétoriques élaborées par les Grecs pour «l'invention», qui représente la première étape de la rédaction d'un discours. De inventione a connu un grand succès comme ouvrage pédagogique pour la rhétorique sous l'Empire romain et au Moyen Âge. La littérature consacrée aux aspects philologiques de ce traité cicéronien est abondante, nous renvoyons à l'excellente synthèse que fait à ce sujet Achard (1994) dans l'introduction à son édition critique du texte latin.

23. Les références sont citées selon l'usage traditionnel philologique que pratiquent les éditions modernes du texte original latin. La traduction de Jean d'Antioche segmente le texte en chapitres (voir ci-dessous), l'extrait analysé correspond aux chapitres VI-IX (p. 87-92 de l'édition Guadagnini).

24. Dans la terminologie rhétorique grecque, on utilise le terme $\sigma \tau \dot{\alpha} \sigma \iota \varsigma$.

25. Le terme est employé ici selon Adam (1990).

26. À propos de l'énumération, voir Jackiewicz (2005), Luc (2001), Pešek (2017), Péry-Woodley et al. (2011).

27. Sur le concept de l'émergence, voir Adam (2012). 
1. la coprésence dans le texte d'au moins deux items disjoints ayant un statut identique par rapport à un élément de référence (un «étalon»);

2. un signal marquant que ces items sont mis en ordre. Ce signalement est réalisé à l'aide de moyens sémiotiques divers: marqueurs lexicaux, chiffres, ponctuation, éléments typographiques, etc. L'effet de série peut être également induit par une simple juxtaposition, pour autant que les items juxtaposés satisfassent à la condition 1 .

En tant que configuration textuelle caractérisée par les deux éléments susmentionnés, la sériation peut être décomposable en plusieurs éléments constitutifs. Une configuration sérielle prototypique comporte toutes ces parties constitutives et représente, du point de vue cognitif, un pôle d'attraction saillant. En même temps, cette configuration prototypique fait office d'outil analytique, par rapport auquel on peut décrire les séries énumératives effectivement réalisées dans les textes.

Voici le modèle prototypique pleinement saturé (cf. Pešek, 2017: 96-97):

\begin{tabular}{|l|l|}
\hline Parties constitutives & Marquage exemple \\
\hline Annonce prospective de la série d'items & Il y a, relativement à X, trois items... \\
\hline \multirow{3}{*}{ Énumération d'items } & Premier item \\
\cline { 2 - 3 } & Deuxième item \\
\cline { 2 - 2 } & Troisième item \\
\hline Clôture rétrospective de la série d'items & Voici ce qu'on peut dire de ces trois items... \\
\hline
\end{tabular}

Tableau 1 - Configuration textuelle de sériation

Comme nous l'avons signalé, les textes authentiques présentent de nombreux écarts par rapport à ce modèle de référence, écarts qui se situent non seulement au niveau structurel (absence d'annonce ou de clôture par exemple), mais aussi au niveau du marquage (absence complète ou partielle de marquage lexical introducteur des items). De plus, les énumérations peuvent comporter des séries enchâssées, car chaque item est susceptible d'être divisé à son tour (tableau 2 ci-après).

L'exposé cicéronien est globalement structuré selon ce modèle sériel enchâssé. Les différents items de la série représentent autant de thèmes qui font partie d'une structure de relations hiérarchisées. L'hyperthème se subdivise en sous-thèmes; dans l'optique présentée ci-dessus, nous dirions que l'hyperthème correspond à l'étalon de référence et les sous-thèmes aux différents items mis en série. L'enchâssement thématique est par endroits très complexe, il peut atteindre jusqu'à sept niveaux de profondeur. Le traité rhétorique cicéronien est donc construit dans une perspective taxinomique mettant en scène les notions (telle, par exemple «l'état de la cause»), leurs divisions et leurs définitions. 


\begin{tabular}{|c|c|c|c|}
\hline Parties constitutives & \multicolumn{3}{|c|}{ Marquage exemple } \\
\hline Annonce & \multicolumn{3}{|c|}{ Il y a, relativement à $X$, trois items... } \\
\hline \multirow{8}{*}{ Énumération } & \multicolumn{3}{|l|}{ Premier item } \\
\hline & \multirow{6}{*}{ Deuxième item $Y$} & \multicolumn{2}{|c|}{ Énumération enchâssée } \\
\hline & & Annonce & $\begin{array}{l}\text { Il y a, relativement à } Y \text {, trois } \\
\text { items... }\end{array}$ \\
\hline & & \multirow{3}{*}{ Énumération } & Premier item \\
\hline & & & Deuxième item \\
\hline & & & Troisième item \\
\hline & & Clôture & $\begin{array}{l}\text { Voici ce qu'on peut dire de ces } \\
\text { trois items... }\end{array}$ \\
\hline & \multicolumn{3}{|l|}{ Troisième item } \\
\hline Clôture & \multicolumn{3}{|c|}{ Voici ce qu'on peut dire de ces trois items... } \\
\hline
\end{tabular}

Tableau 2 - Séries enchâssées

\subsection{Structure compositionnelle et thématique de l'extrait}

Ci-dessous (figures 1 et 2), nous donnons une représentation schématique de l'extrait analysé qui est, comme nous venons de le dire, organisé selon le mode sériel.

\section{constitucion}

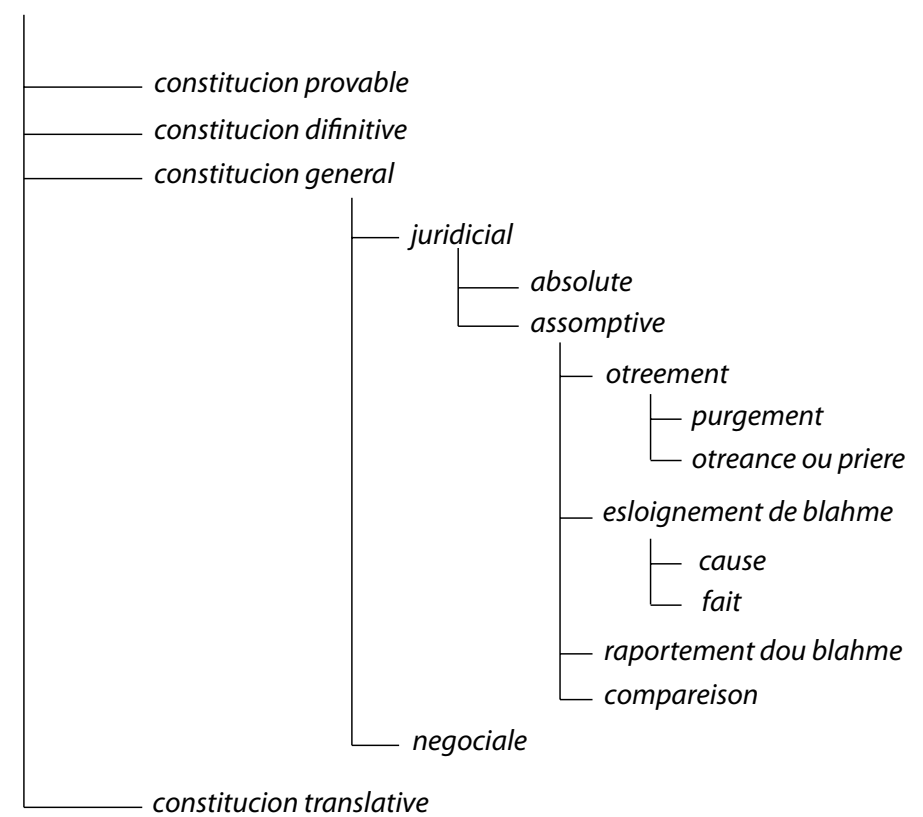

Figure 1 - Hiérarchie notionnelle constitucion 


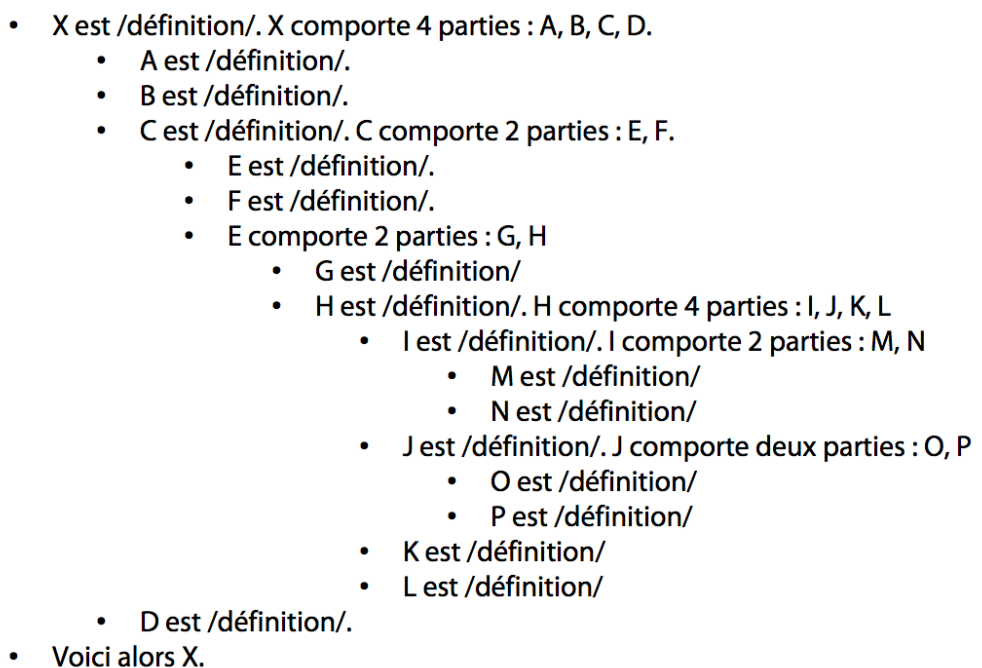

Figure 2 - Structure compositionnelle de l'extrait (I, 10-16)

La figure 1 présente la hiérarchie notionnelle dominée par le terme constitucion (= état de la cause, voir ci-dessus), elle montre par là même la terminologie rhétorique introduite par Jean d'Antioche.

La figure 2 schématise la structure compositionnelle de l'extrait, les lettres d'alphabet correspondent aux différentes notions de la figure 1 ( $\mathrm{X}$ = constitucion, $\mathrm{A}=$ constitucion provable, $\mathrm{B}=$ constitucion difinitive et ainsi de suite). Il s'agit d'une configuration sérielle enchâssée; l'enchâssement atteint six niveaux.

Comme on le voit, les séries présentent une organisation fortement prototypique. Elles sont ouvertes par une phrase d'annonce qui énumère les items, voir les exemples suivants [1]-[4] ${ }^{28}$ :

[1] Ceste constitucion donques que nos apelons «general» [C] ${ }^{29}$ si a .ii. parties, ce m'est avis: la juridiciale $[\mathrm{E}]$ et la negocial $[\mathrm{F}]$.

Suit la présentation des différents items:

[2] La juridiciale [E] si est en la quele est demandee et quise la nature de droit et d'igalité ou la raison de guerredon ou de paine [...].

28. Pour faciliter la lecture de ces exemples illustratifs, nous joignons ici la traduction en français moderne. Elle est basée sur l'usage d'Achard $(1994: 69,71)$. [1] Donc cet état que nous appelons de qualification nous semble avoir deux parties: l'équitable et la légale. [2] L'équitable est celle où l'on recherche quelle est la nature $d u$ juste et du bien, quels sont les principes d'attribution des récompenses et des châtiments [...]. [3] La légale est celle où l'on recherche ce qui est conforme au droit d'après les usages de la cité et d'après l'équité: ce dont s'occupent chez nous les sages et les jurisconsultes. [4] Nous venons d'exposer et d'indiquer les états de cause et leurs parties $[. .$.$] .$

29. Entre crochets, nous mettons la lettre qui réfère aux structures représentées sur la figure 2. 
[3] La negociale [F] si est en la quele l'on regarde quel droit ou quel chose de droit soit de la droiture et de la coustume civile, sur le quel esgart ou dilegence chiez nos et selonc nostre usage doivent estre les sages et les conseilleors de droit.

La série enchâssante supérieure (constitucion) comporte également une phrase de clôture:

[4] Nos avons espons et esclarsi les constitucions [X] et lor parties [...].

Du point de vue de la structure informationnelle, la partie thématique des phrases d'annonce contient la notion hiérarchiquement supérieure. Dans la partie rhématique est introduite la première mention des items, qui représentent des divisions notionnelles de la notion supérieure (voir l'exemple [1]). Par la suite, les différents items sont repris par la voie de la progression linéaire et figurent ainsi dans la partie thématique des énoncés subséquents (voir les exemples [2] et [3]).

Nous venons de décrire la structure compositionnelle et thématique de l'extrait. Conformément à notre objectif, nous allons maintenant mettre cette structure en corrélation avec le marquage typographique de l'édition moderne d'une part et du manuscrit original de l'autre part. Nous commencerons par l'analyse de l'édition moderne, car celle-ci met en œuvre le dispositif typographique habituel. Les énoncés, les phrases complexes, les paragraphes et les chapitres sont ici des réalités typographiques de la structure du texte. Il est par conséquent possible d'appliquer à l'analyse les principes et les catégories discutés dans la partie 2. Ces éléments sont en revanche inexistants dans le manuscrit, qui utilise d'autres marques typographiques pour délimiter les segments textuels pertinents. La comparaison des deux systèmes permet ainsi d'illustrer le fonctionnement de la typographie considérée du point de vue de la structure thématique et compositionnelle du texte.

\subsection{L'édition moderne de Guadagnini - division en paragraphes et structure compositionnelle}

Faisons tout d'abord remarquer que l'édition moderne garde la division en chapitres qu'a établie Jean d'Antioche et qui est donc celle du manuscrit (voir ci-dessous). Quant à la division en paragraphes, elle est opérée par l'éditrice du texte et elle n'est motivée par aucun signe typographique du manuscrit. La corrélation paragraphes/structure thématique et compositionnelle est représentée par le schéma suivant (figure 3). Le schéma précise d'abord la division en chapitres (colonne de gauche «Chapitres») ${ }^{30}$. Ensuite nous marquons, relativement à chacun des chapitres, la division en paragraphes (colonne «Paragraphes»). Les colonnes «(hyper)thème

30. On remarque que dans le schéma, il manque le chapitre VII. En effet, ce chapitre est une digression dans le cadre de laquelle Cicéron présente la division que fait Hermagoras de la constitutio generalis. Cicéron critique et rejette cette division d'Hermagoras. La digression rompt le cours de la série énumérative /constitucion/ qui reprend comme si de rien n'était dans le chapitre VIII. Dans la version de Jean d'Antioche, la reprise est marquée par un donques (Ceste constitucion donques que nos apelons "general» [...]) qui signale que les arguments d'Hermagoras ont été réfutés et que l'exposé reprend là où il s'est arrêté (en latin, il 
niveau 1-6» spécifient la structuration thématique et compositionnelle relativement aux paragraphes, les chiffres 1-6 indiquent le niveau d'enchâssement thématique et sériel. Les lettres d'alphabet réfèrent aux éléments notionnels spécifiés sur la figure 2 (voir aussi la note 29). Étant donné la nature du texte, ces éléments représentent des nœuds thématico-rhématiques de la structure informationnelle de l'extrait analysé.

En considérant les propriétés thématiques des différents paragraphes, nous constatons une certaine diversité. Pour la décrire, nous allons utiliser la terminologie qu'introduit Daneš dans son étude de 1994, que nous avons présentée ci-dessus ${ }^{31}$.

Dans l'extrait analysé, il y a d'un côté des paragraphes qui ne concernent qu'un seul élément notionnel: $§ 2,3,4,8,9,10$. Dans ce cas, les thèmes des énoncés sont tous identiques et correspondent au thème du paragraphe: ces paragraphes sont thématiquement homogènes. Certains de ces paragraphes $(2,3,4)$ contiennent une exemplification; le type de l'état de la cause présenté dans le cadre du paragraphe est illustré par un cas concret ${ }^{32}$.

D'un autre côté, il y a des paragraphes dont les énoncés contiennent des thèmes différents $(\S 1,5,6,7)$, le thème de paragraphe se voit divisé en sous-thèmes qui deviennent à leur tour des thèmes d'énoncé. Dans le cadre de cette configuration, on peut distinguer deux cas de figure: soit le thème de paragraphe est unique et correspond à une seule notion d'un niveau hiérarchique donné $(\S 1,5,6)$, soit le thème de paragraphe est double, car il correspond à deux notions d'un même niveau hiérarchique $(\S 7)$. Ainsi dans le $\S 5$, le thème de paragraphe est représenté par la notion constitucion general. Cette notion-thème (niveau 2 d'enchâssement thématique) est ensuite subdivisée en 2 éléments - constitucion general juridicial et constitucion general negociale - qui représentent eux-mêmes des thèmes d'énoncé (niveau 3 d'enchâssement). Dans le $\S 7$, il y a deux thèmes de paragraphe - otreement et esloignement de blabme - qui se situent au même niveau d'enchâssement (niveau 5). Ces deux thèmes développent chacun leur propre embranchement thématique (niveau 6 d'enchâssement), mais toujours dans le cadre d'un seul paragraphe.

Selon la typologie danešienne, les $\S 2,3,4,8,9,10$ correspondent au type I. «thème stable», les $\S 1,5,6$ au type II. 1 «thématisation des aspects du thème de paragraphe» et le paragraphe 7 au type II. 2 «éclatement thématique» ${ }^{33}$.

y a un ergo, la traduction moderne opte également pour un donc). Nous la traitons à part comme une sorte de parenthèse qui s'insère dans le texte, elle ne fait pas partie de la série énumérative.

31. Rappelons que Daneš, dans sa terminologie, distingue le thème de paragraphe (O-thème, odstavcové téma) qui est une sorte d'hyperthème qui domine le paragraphe, et le thème d'énoncé (V-thème, výpovědní téma) qui a par définition un rapport au thème de paragraphe.

32. Dans la version de Jean d'Antioche, cette exemplification est introduite par la tournure si come se. En latin, Cicéron utilise un $u t$ et la version moderne opte pour l'organisateur par exemple. Comme cette exemplification concerne le thème de paragraphe, nous considérons ces paragraphes comme thématiquement homogènes.

33. Thème stable: stabilní O-téma; aspectualisation thématique: tematizace aspektů O-tématu; clivage thématique : rozštěpení $O$-tématu. 


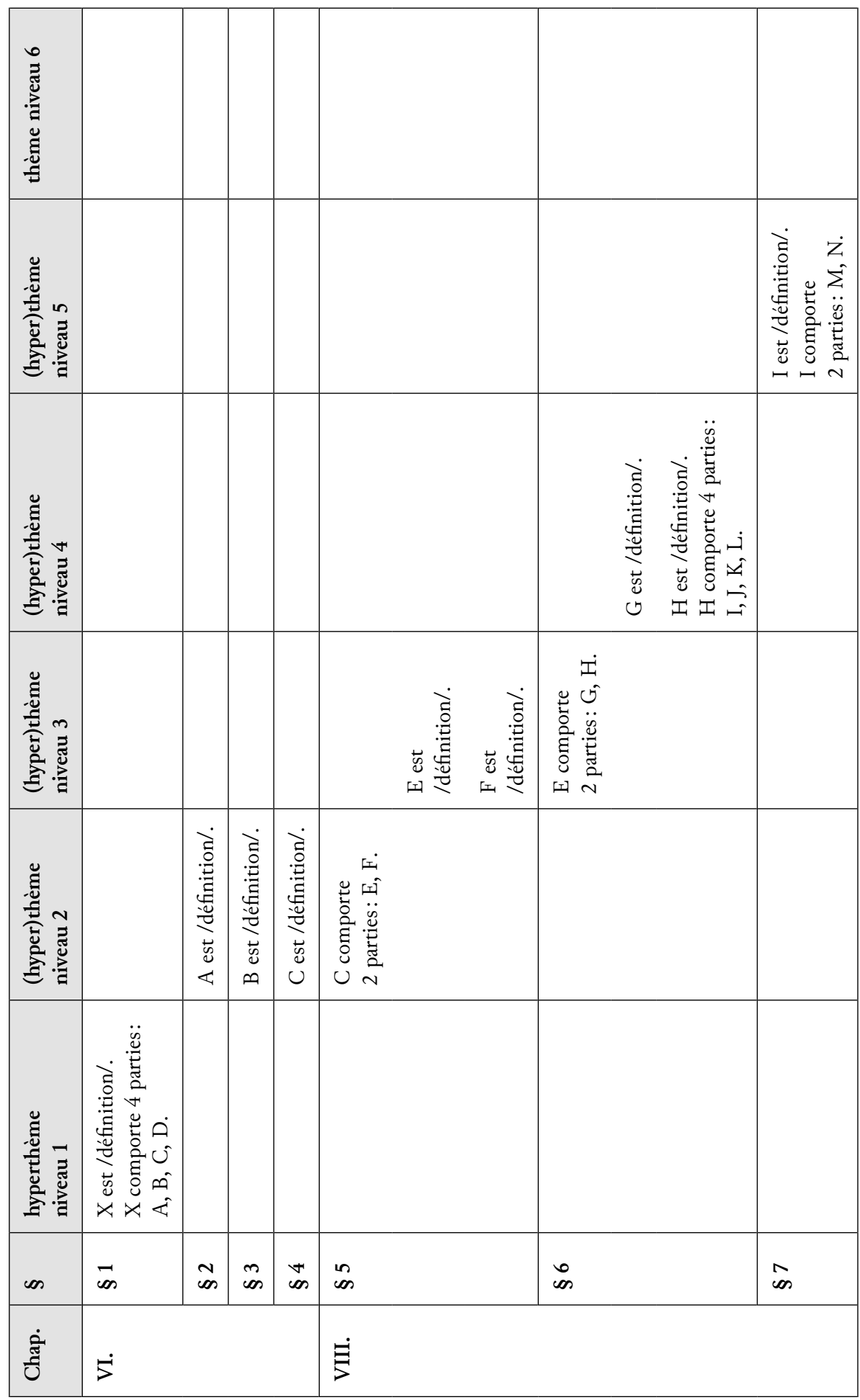

URL: http://journals.openedition.org/discours/10794 


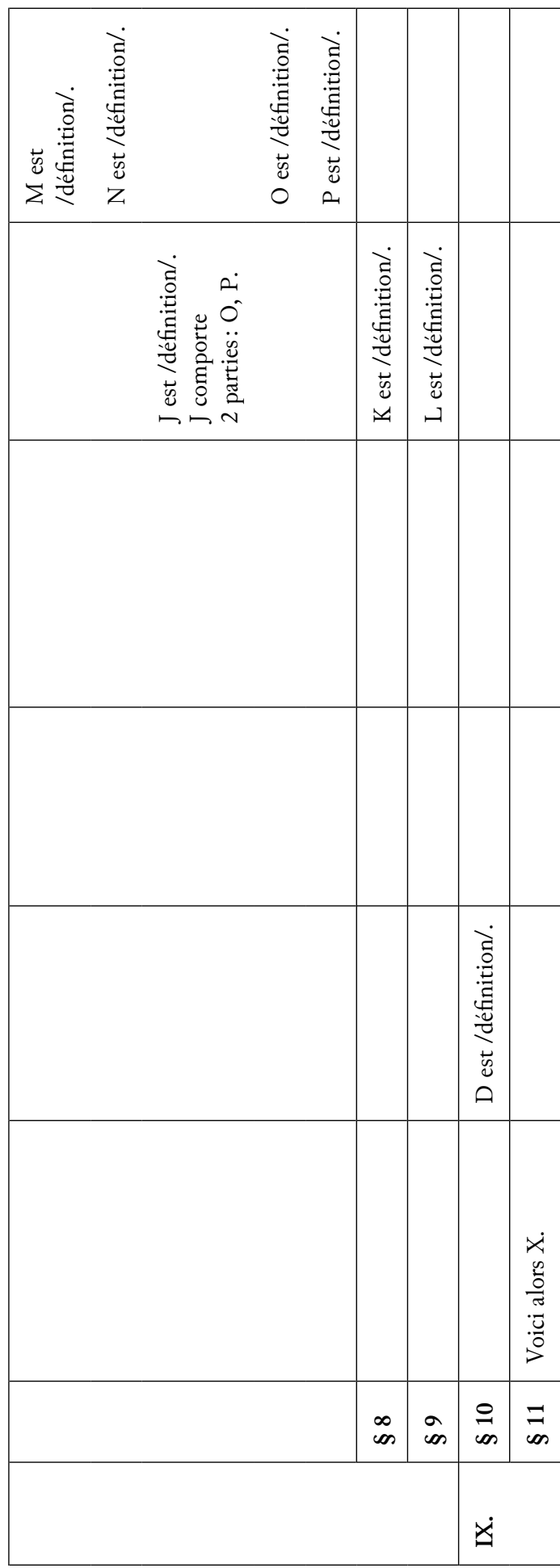

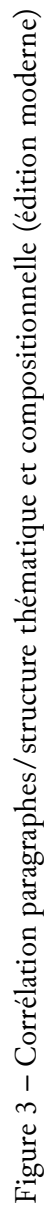


Si nous considérons la segmentation en paragraphes du point de vue compositionnel (mode sériel, voir ci-dessus), nous constatons les faits suivants. Le traitement d'un item (définition, explication, exemple) est toujours confiné au sein d'un seul paragraphe. Quant aux phrases d'annonce, elles figurent soit à la fin du paragraphe, dans ce cas, le paragraphe subséquent commence par la présentation du premier item $(\S 1-2, \S 6-7)$, soit au début du paragraphe, qui contient par là même la présentation des items (§6).

Quant aux relations thématiques entre paragraphes, considérées du point de vue de leurs rapports hiérarchiques, nous constatons que ce n'est pas tant la position dans la hiérarchie thématique qui gouverne la segmentation en paragraphes, mais plutôt la longueur du segment qui traite d'un thème donné. Ainsi le $\S 7$ contient plusieurs thèmes de niveaux différents, alors que les $\S 8$ et 9 correspondent chacun à un seul thème, du même niveau que ceux traités dans le cadre du paragraphe précédent. Si les deux thèmes (K et L) étaient incorporés au $\S 7$, ensemble avec les autres membres du même niveau thématique (I et J), ce paragraphe en deviendrait trop long par rapport aux autres paragraphes du texte.

Bien que la structuration thématique et compositionnelle des paragraphes soit très hétérogène, nous pouvons constater que c'est le principe thématique qui détermine d'une manière décisive la segmentation opérée. En effet, les confins de chaque paragraphe correspondent au début ou à la fin d'une section thématique sans égard au niveau d'enchâssement. Les phrases d'annonce font figure de transition thématique. Annonçant de nouveaux thèmes dérivés du thème précédent, elles peuvent se trouver à la fin ou au début du paragraphe.

\subsection{Le manuscrit - marques typographiques et structure compositionnelle et thématique}

Les mêmes principes selon lesquels nous avons analysé l'édition moderne seront appliqués à l'analyse du manuscrit, les marques typographiques utilisées par le scribe seront corrélées avec les parties constitutives de la structure compositionnelle et thématique du texte.

Comme on le sait, la pratique typographique des documents manuscrits médiévaux n'a pas été unifiée, ni quant aux signes utilisés ni quant aux principes d'usage de ces signes. Tout au plus peut-on constater des tendances ou des habitudes qui représentent une sorte de fonds commun, mais malgré ces éléments communs, chaque manuscrit demeure singulier et doit être traité à part:

Chaque scribe établit son propre sous-système, sa propre distribution de valeurs; [...] cela se traduit en une large polyvalence des signes; [...] le scribe n'applique pas toujours à l'intérieur d'un même codex les critères suivis dans une section déterminée.

(Llamas Pombo, 2001: 153)

En dépit de cette diversité, l'usage des marques de ponctuation n'est jamais gratuit. Comme le souligne Lavrentiev (2016: 42): 
Les marques de ponctuation n'apparaissent jamais (ou presque) dans des endroits où leur usage ne peut être expliqué par un facteur ou un autre, mais ces facteurs sont multiples et hétérogènes: des constructions syntaxiques, des mots particuliers «attirant» la ponctuation, le changement de plan énonciatif, la mise en relief de noms propres ou de personnages importants ou encore la décoration de la page manuscrite.

Dans le manuscrit de la Rectorique (voir ci-dessus, section 3.1), nous trouvons les signes typographiques suivants (la figure 4 montre des exemples concrets d'usage de ces signes dans le manuscrit):

- point placé à mi-hauteur de la dernière lettre du mot $^{34}$;

- virgule - dans notre manuscrit, il s'agit d'une barre oblique qui commence à mi-hauteur de la ligne et qui est orientée vers le haut de la ligne ${ }^{35}$;

- majuscule rubriquée;

- pied de mouche;

- lettrine initiale colorée - dans ce cas, il faut distinguer les premières lettres de chapitre, qui sont richement colorées et décorées, des lettrines intérieures, qui sont plus petites et moins décorées que les initiales de chapitre;

- intitulés de chapitres rubriqués.

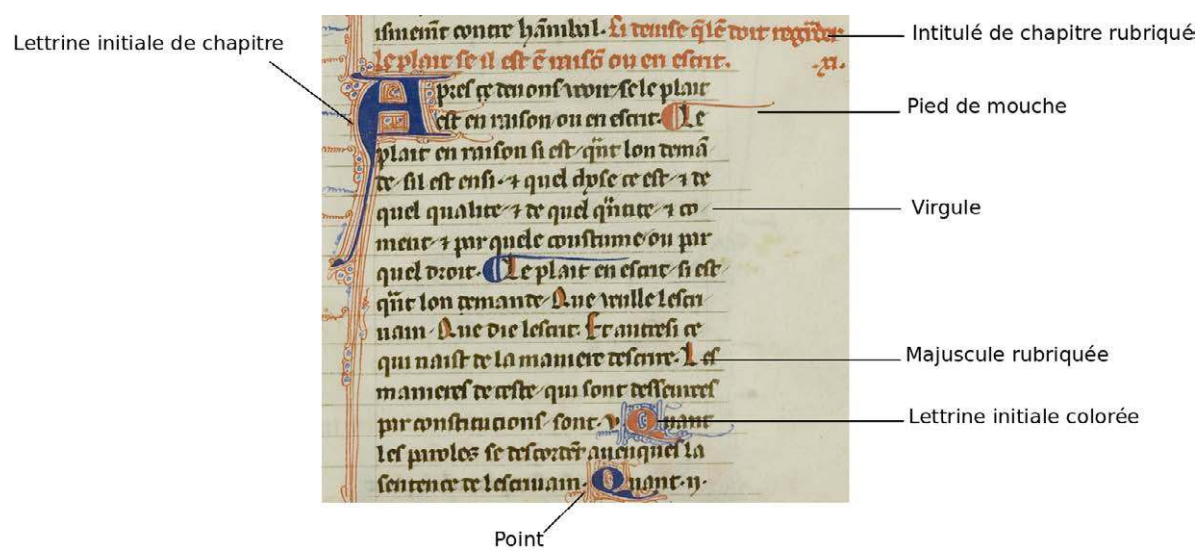

Figure 4 - Exemples : manuscrit (chapitre IX)

34. Nous préférons ici utiliser le terme simple "point». Arabyan (2012: 204) appelle le point à mi-hauteur de la dernière lettre du mot «colon», suivant ainsi la division tripartie introduite par Isidore de Séville (periodus, colon, comma). Pour autant, cette dénomination n'a de sens que si le système tripartite est complet. Puisque ce n'est pas le cas de notre manuscrit, nous utilisons le terme de «point».

35. De même que dans le cas du «point», nous utilisons le terme «virgule» et non pas «comma». D'abord, le système qu'utilise le manuscrit n'est pas tripartite (voir note 34), mais aussi, la forme du signe est particulière (il n'y a pas de point). 
Le fonctionnement complexe de ces signes met en jeu plusieurs facteurs de natures diverses. Notre analyse procédera en deux phases. D'abord, nous décrirons les conditions d'apparition des différents signes en tenant compte de la variété des contextes. Ensuite, dans une perspective synthétique, nous essaierons de proposer une typologie des fonctions que peuvent porter les signes du manuscrit analysé. Ce faisant, nous prêterons une attention particulière aux éléments du marquage de la structuration textuelle.

\subsubsection{Point et majuscule}

Nous constatons une forte corrélation entre les points et les majuscules rubriquées. Toutes les majuscules sont précédées d'un point, mais les points peuvent également apparaittre devant une minuscule non rubriquée. Contrairement à l'usage moderne, la majuscule ne sert pas à marquer les noms propres. Du point de vue syntaxique, les segments délimités par le point + majuscule correspondent à des entités diverses. Dans la majorité des cas, cette séquence typographique délimite une proposition principale ${ }^{36}$.

Les conjonctions de coordination, typiquement les et, mais, ou et car, sont également précédées d'un point et écrites avec une majuscule initiale ${ }^{37}$, de même que les phrases qui contiennent un déterminant relatif ${ }^{38}$. Nous constatons donc que la pratique typographique de notre manuscrit ne s'accorde point avec la conception moderne de la phrase. Le marquage employé (point + majuscule) estompe la différence entre la juxtaposition (marquée aujourd'hui par une virgule ou un pointvirgule + minuscule) ou la coordination (marquée aujourd'hui par une conjonction de coordination écrite avec une minuscule et précédée éventuellement d'une virgule) d'une part et le lien dit «textuel» (marqué aujourd'hui par un point + majuscule) d'autre part. L'exemple suivant montre la différence entre la ponctuation utilisée dans le manuscrit et la ponctuation utilisée dans l'édition moderne (Guadagnini):

Manuscrit ${ }^{39}$ :

Nos avons espons et esclarsi les constitucions et lor parties - Desoresmais dirons les exemples de chascune maniere - Mais il est avis/ que quant nos donrons abondance d'argumens en chascun des eissamples/ de tant les expondrons et les esclarsirons

36. Nous entendons par là une proposition (portée par la relation sujet + verbe conjugué) qui ne dépend d'aucune autre proposition en tant que subordonnée syntaxique.

37. Il est toutefois possible de trouver des exceptions à ce principe syntaxique, car rarement nous trouvons des cas où la conjonction et qui fonctionne comme un coordonnant phrastique est précédée d'un point, mais écrite avec une minuscule (I, 9; p. 87, ligne 15 de l'édition Guadagnini).

38. Ces structures sont plutôt rares en français moderne. Ex.: Le plait dou num si est/ quant l'on parle dou fait et preuve/ et l'on demande ce qui est fait/par quel num/ sera apelé. En la quel maniere de constitucion/ il est mestier/ que la controversie ou le plait soit del num [...]. La traduction d'Achard opte pour un démonstratif: au segment en la quel maniere de constitucion qui traduit la structure latine quo in genere (structure relative) correspond le syntagme dans ce genre de cause de la traduction moderne (I, 11).

39. Dans notre transcription de la ponctuation du manuscrit, nous marquons les points $\cdot$, les virgules / et les majuscules colorées D. 
plus profitablement - Car la raison d'argumenter sera plus clere/ quant ele se porra maintenant afaitier a la maniere et a l'eissample de la cause.

Édition moderne (Guadagnini):

Nos avons espons et esclarsi les constitucions et lor parties: desoresmais dirons les exemples de chascune maniere; mais il est avis que quant nos donrons abondance d'argumens en chascun des eissamples de tant les expondrons et les esclarsirons plus profitablement, car la raison d'argumenter sera plus clere quant ele se porra maintenant afaitier a la maniere et a l'eissample de la cause.

Nous pouvons ainsi dire que le texte ancien ne connait pas de segments du type «phrase complexe» composés de plusieurs propositions principales. Ce constat rejoint les arguments qu'avancent certains chercheurs contemporains lorsqu'ils problématisent la notion de "phrase» (voir Groupe de Fribourg, 2012, ou Combettes, 1998): en dernier ressort, la définition de la phrase est une définition typographique.

La séquence typographique point + majuscule n'est pas réservée qu'aux segments du type phrastique. Elle est également utilisée pour marquer une suite de syntagmes nominaux saillants, qu'ils soient introduits par une conjonction de coordination ou non.

\subsubsection{Virgule}

L'usage de la virgule est quantitativement très élevé dans notre manuscrit. Systématiquement, elle précède les conjonctions de subordination et les pronoms relatifs, elle délimite également la subordonnée antéposée de la principale. Elle peut aussi séparer des syntagmes nominaux ou prépositionnels à l'intérieur de la phrase. D'une manière très irrégulière, la virgule figure dans les structures avec si dans lesquelles le $\mathrm{SN}$ représente le topique externalisé ${ }^{40}$.

Enfin, la virgule est utilisée pour marquer la division d'un mot à la fin de la ligne.

\subsubsection{Intitulés de chapitre, pieds de mouche et lettrines colorées}

Malgré la diversité d'usage, nous pouvons dire que la séquence point + majuscule et la virgule opèrent plutôt au niveau micro-textuel. En revanche, les lettrines colorées, les pieds de mouche et les intitulés de chapitre rubriqués peuvent marquer des segments qui représentent des unités compositionnelles du texte aux niveaux méso- ou macro-textuel (voir Adam, 2018: 84). Sur la figure 5, qui est analogue à la figure 3 , nous représentons la corrélation entre les éléments de la structure compositionnelle (schématisée sur la figure 2) et les marques typographiques du manuscrit. Celles-ci correspondent aux signes suivants utilisés dans le schéma: $I$ - lettrine initiale colorée (la lettrine de chapitre est «plus grande»), II - pied de mouche, VIII. - chapitres rubriqués. Les couleurs correspondent elles aussi au manuscrit (dans le cas des lettrines et des pieds de mouche, le manuscrit fait alterner le rouge et le bleu).

40. Ex.: Constitucion/ si est le premier debat des causes ou des plais/ qui vient dou deboutement de l'entencion [...] (voir Pešek, 2017). 


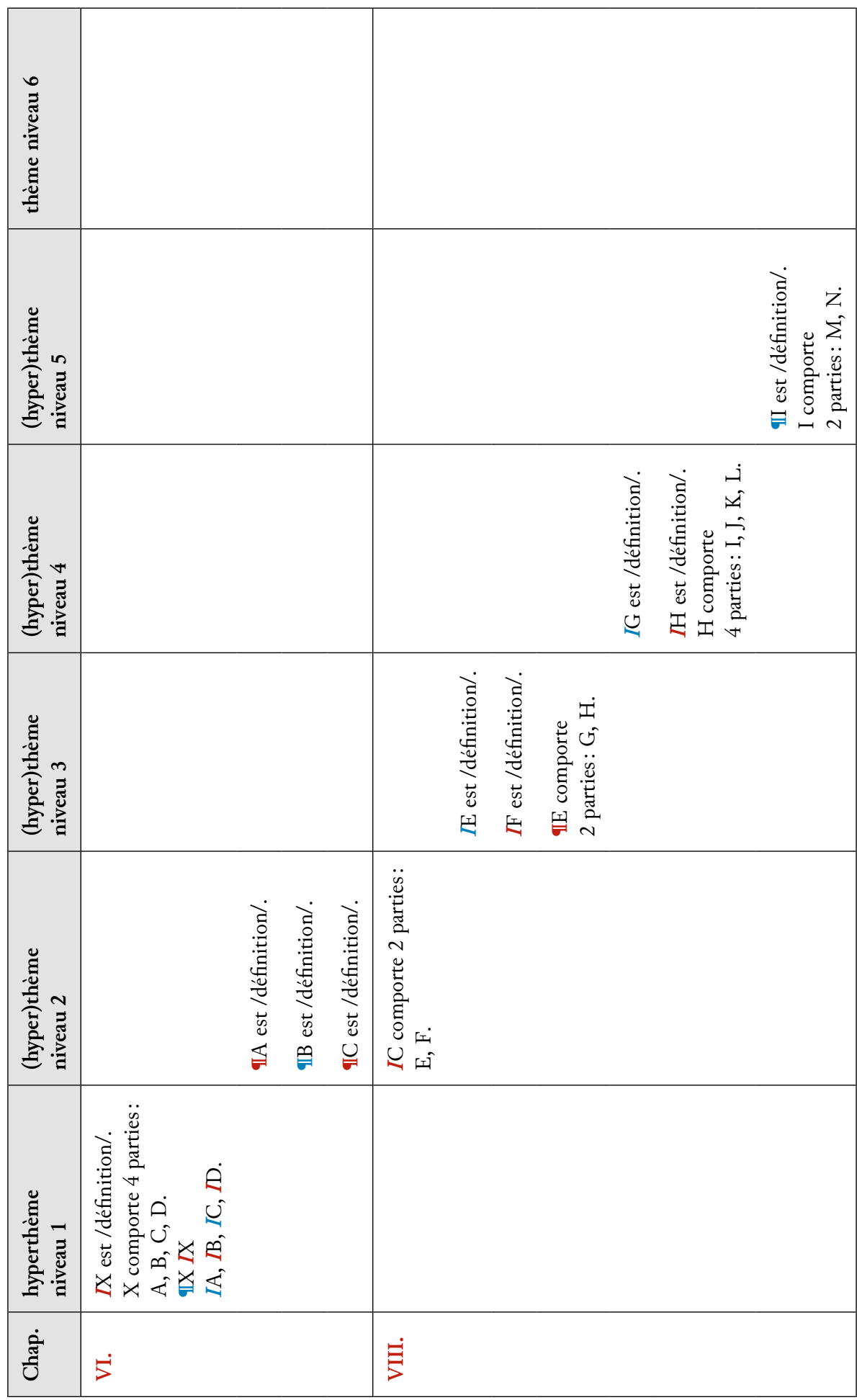

URL: http://journals.openedition.org/discours/10794 


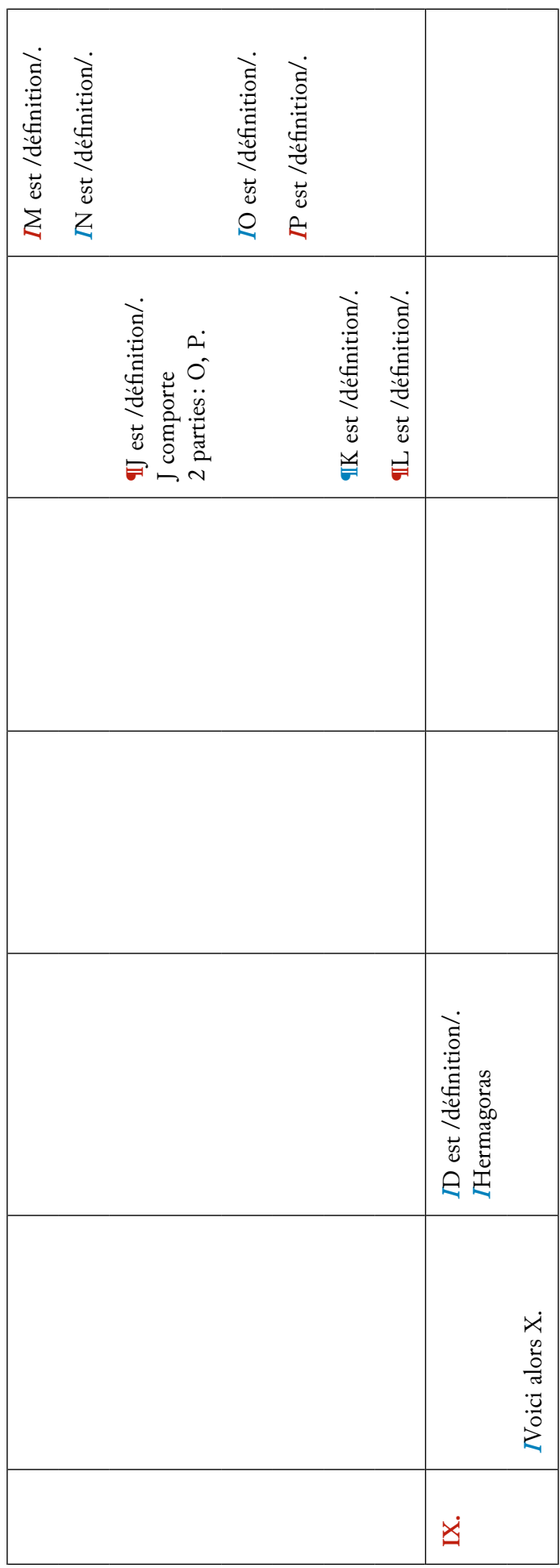

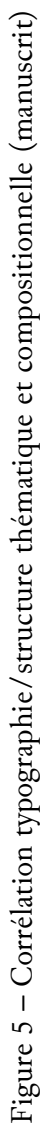




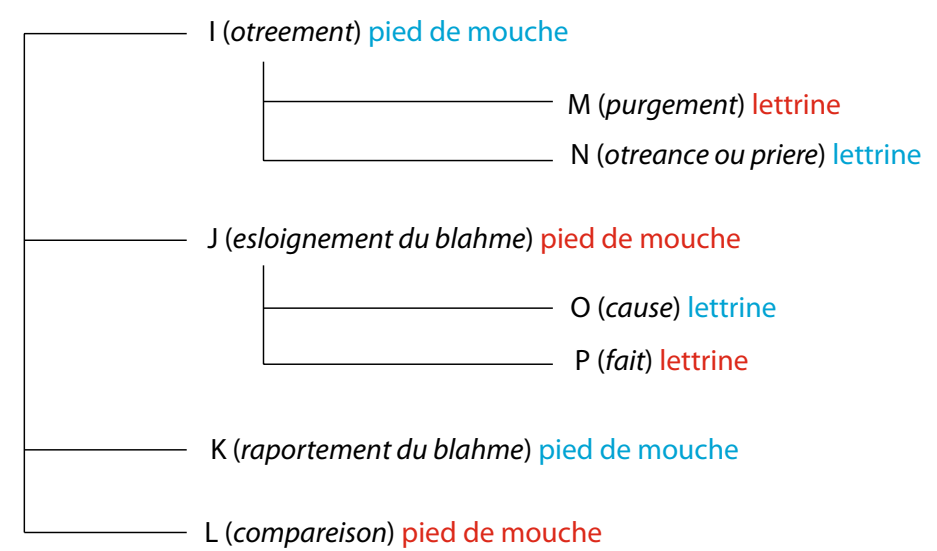

Figure 6 - Structure compositionnelle et thématique: exemple du marquage (chapitre VIII)

Nous constatons que dans l'extrait analysé, ces marques apparaissent à la charnière des unités compositionnelles du texte. De plus, elles ont des rapports hiérarchiques. Le niveau supérieur est marqué par les intitulés des chapitres ${ }^{41}$. Ensuite viennent les pieds de mouche; les lettrines colorées se situent au niveau inférieur de la hiérarchie. Autrement dit, le texte entier est segmenté en chapitres ${ }^{42}$, les chapitres sont segmentés par les pieds de mouche et les lettrines colorées marquent des segments au sein des segments délimités par les pieds de mouche. Du point de vue compositionnel, les pieds de mouche et les lettrines délimitent les sections thématiques qui correspondent aux différentes composantes de la configuration sérielle. Les éléments thématiques du même niveau sont délimités par le même signe, la hiérarchie pied de mouche/lettrine étant observée. Ainsi, dans le chapitre VIII, les éléments des niveaux hiérarchiques 5 et 6 sont marqués comme dans la figure 6 .

Les segments délimités par les pieds de mouche ${ }^{43}$ sont d'envergure différente, mais contiennent typiquement plusieurs segments délimités par point + majuscule (et éventuellement point + lettrine colorée).

41. Le chapitre n'est chapitre qu'en vertu du marquage de la segmentation. Sans intitulé rubriqué, il n'y a pas de chapitre. Formellement, les chapitres sont marqués par l'encre rouge, la première lettre du chapitre est richement colorée et ornée.

42. Pour être précis, le texte est segmenté en livres et les livres en chapitres. Le début d'un livre est simplement annoncé dans l'intitulé du chapitre: Ci comense le segont livre de Rectorique de Marc Tulles Cyceron. .xxxviii. Comme notre analyse ne concerne qu'un seul extrait, la segmentation en livre n'entre pas directement dans notre analyse. Pour plus d'information à ce sujet, voir Pešek (2017).

43. Remarquons un fait intéressant. Dans la pratique moderne, la notion de paragraphe est utilisée pour désigner et la forme (même si dans ce cas on parle parfois de l'alinéa) et le segment même. Pour le manuscrit, la marque formelle est le pied de mouche, mais comment appeler le segment? Utiliser le terme de paragraphe serait pour nous un anachronisme, étant donné également que dans le manuscrit il y a d'autres signes qui peuvent être considérés comme délimitant les "paragraphes», telles les lettrines. 
Quant aux lettrines colorées, il faut souligner qu'elles ont d'autres fonctions que celle de segmenter: elles marquent un élément notionnel saillant et, naturellement, elles embellissent le texte. Il serait même possible de se demander si la fonction segmentatrice n'est pas que secondaire et «dérivée», car elle entre en jeu lorsque cet élément notionnel saillant occupe la partie thématique de l'énoncé; il s'agit typiquement des configurations sérielles dans lesquelles les items, introduits dans une phrase d'annonce, apparaissent par la suite en tant que thèmes-topiques pour être expliqués et définis. Tout de même, la lettrine colorée n'est pas réservée aux éléments notionnels: par exemple dans le chapitre IX (I, 16) sont marqués les éléments Hermagoras et Nos qui n'ont aucun rôle dans le système notionnel du texte cicéronien. L'endroit où apparaît ce marquage correspond aux confins d'une unité thématiquement homogène et segmentable à l'instar des paragraphes modernes. Nous avons donc décidé de considérer les lettrines colorées comme des marques de segmentation textuelle, tout en insistant sur leur polyfonctionnalité ${ }^{44}$.

La segmentation au niveau macro- et méso-textuel que pratique le manuscrit peut donc être considérée comme tripartite (chapitre/pieds de mouche/lettrines), à la différence des éditions modernes qui emploient une segmentation bipartite en chapitres et en paragraphes.

\subsubsection{Synthèse}

Nous sommes certes conscients du danger qui consiste à vouloir corréler nos catégories modernes avec les signes typographiques anciens. Cela ne nous empêche pas pour autant de catégoriser le fonctionnement de ces signes. Précisons bien une chose: les catégories fonctionnelles que nous postulons (textuelles, syntaxiques, esthétiques...) servent à saisir ces signes dans le contexte de la linguistique contemporaine, mais elles ne peuvent pas être instrumentalisées pour expliquer la motivation qu'eut le copiste médiéval pour mettre tel ou tel signe à tel ou tel endroit.

Voici donc en résumé les différentes fonctions de la typographie du manuscrit. Naturellement, un seul signe peut relever de plusieurs catégories fonctionnelles en même temps.

$1^{\text {re }}$ fonction: segmentation textuelle - nous insistons beaucoup sur l'épithète «textuelle», car les segments délimités peuvent être analysés comme des paquets de propositions-énoncés d'une complexité croissante.

- Chapitre (typographiquement: intitulé résomptif rubriqué, lettrine initiale amplement colorée).

- Pied de mouche: délimite des paquets de propositions-énoncés d'une envergure variable au sein du chapitre ${ }^{45}$.

44. La fonction segmentatrice des lettrines est d'ailleurs mentionnée dans Englebert (2000) et dans Llamas Pombo (2001).

45. On fait souvent remarquer que les pieds de mouche sont des précurseurs typographiques des alinéas (paragraphes), voir Arabyan (2012). 
- Lettrine colorée: délimite des paquets de propositions-énoncés d'une envergure variable au sein des pieds de mouche ou directement au sein du chapitre.

- Point + majuscule rubriquée: délimite des phrases principales autonomes, des propositions-énoncés de base.

- Point + minuscule : certains cas de coordination syntaxique (les segments représentent des propositions-énoncés).

$2^{\mathrm{e}}$ fonction: segmentation syntaxique intrapropositionnelle.

- Virgule (syntagmes nominaux, syntagmes prépositionnels, propositions subordonnées ${ }^{46}$, etc.).

$3^{\mathrm{e}}$ fonction: marquage des éléments notionnels saillants.

- Lettrine colorée.

- Point + majuscule rubriquée.

$4^{e}$ fonction: Indice de lien des mots séparés en fin de ligne.

- Virgule.

\section{$5^{\mathrm{e}}$ fonction: Esthétique.}

- Lettrine initiale de chapitre.

- Lettrine colorée.

- Majuscule rubriquée.

- Intitulé de chapitre rubriqué.

\section{Conclusions - partielles et générales}

L'étude de cas que nous venons de présenter nous permet de formuler pour commencer quelques conclusions partielles. D'abord, l'analyse a confirmé l'hypothèse qui dit que la structure textuelle matérielle (Jacques, 2005; Luc et Virbel, 2001) a pour but de faciliter la vi-lisibilité des unités du plan du texte. Chapitres, paragraphes, pieds de mouche, lettrines: tous ces éléments délimitent des segments qui correspondent aux parties de la structure compositionnelle et thématique du texte. L'envergure des unités délimitées peut certes varier, mais c'est toujours le principe sémantique textuel qui gouverne la segmentation opérée. L'analyse de la pratique typographique du manuscrit s'est montrée très pertinente à cet égard. Nous avons vu en effet

46. L'on sait que certaines subordonnées peuvent fonctionner comme des propositions-énoncés autonomes (certaines causales, les concessives et autres). La virgule aurait dans ce cas une fonction de segmentation «textuelle». Étant donné que la frontière entre ces deux catégories est floue, nous simplifions la situation en rangeant les subordonnées dans la catégorie «intrapropositionnelle», la subordonnée étant considérée comme une partie de la proposition principale. 
qu'au niveau micro-textuel (propositions-énoncés, périodes), la segmentation diffère fondamentalement de la pratique moderne. S'il est possible de reconstituer ces unités sur la base sémantique (rhétorique) et de refléter cette reconstitution par la ponctuation moderne dans les éditions contemporaines, la typographie du manuscrit n'est pas guidée par ce principe et segmente les unités autrement. Le concept de la «phrase» que nous transmet notre tradition linguistique se trouve fortement ébranlé. Malgré les différences au niveau micro-textuel, la typographie méso-textuelle (paragraphes, pieds de mouche, lettrines) et macro-textuelle (chapitres) poursuit un but identique: rendre le texte vi-lisible en proposant une grille de lecture segmentée selon le principe compositionnel et thématique.

Pour les conclusions générales, nous commencerons par dire que le paragraphe, en tant que dispositif de la matérialité textuelle, est (i) une division, un segment sinon une unité de composition textuelle, (ii) graphique sinon strictement sémantique...

Toute la difficulté réside dans le fait qu'il n'est pas réductible à une "grammaire générative», pas plus d'ailleurs que ne l'est le texte depuis que celui-ci et ses divisions (ses unités constitutives) font l'objet de l'analyse:

L'analyse du texte préalablement segmenté [notamment en paragraphes] aurait pu être prolongée par une démarche constructive, cherchant à donner une représentation logico-sémantique, plus ou moins formalisée, de cet objet sémiotique, et à proposer un simulacre de sa production. Nous ne l'avons pas fait, pour des raisons théoriques d'abord: le statut sémiotique d'une telle construction ne serait nullement assuré, à défaut d'une grammaire discursive capable de générer des textes-objets... (Greimas, 1976: 264)

Cela ne veut pas dire qu'il faille renoncer à l'analyse linguistique des textes et de ses segments (composants ou unités).

Ainsi, nous plaçant à la suite de Daneš et tout récemment d'Adam, la question que nous nous sommes posée est la suivante: l'analyse du paragraphe (et des segments matériels analogues) en termes de progressions (relations et ruptures) thématiques, faciliterait-elle alors la tâche de sa définition (délimitation)? Autrement dit: la prise en compte de l'analyse thématique est-elle pertinente pour l'analyse du paragraphe comme unité (constitutive) du texte? Nous pensons avoir répondu par l'affirmative: dans le texte analysé du moins, la pertinence de la division en paragraphes se déduit fondamentalement de la structure thématique et compositionnelle. Les frontières de ces segments correspondent aux confins des segments thématiques qui, eux, sont en même temps constitutifs de la structure compositionnelle (configuration sérielle) du texte. Néanmoins, cette relation n'est pas biunivoque : étant donné que le paragraphe est une entité linéaire et "plate», il n'est pas en mesure de refléter l'aspect hiérarchique de la structure thématique. Cette hiérarchie, si l'on veut, doit être marquée par d'autres types de signaux (lexicaux ou typographiques). À cet égard toutefois, le marquage du manuscrit médiéval est 
"plus puissant», car, comme on l'a vu, l'usage des éléments marqueurs (pieds de mouche, lettrines colorées) reflétait, ne serait-ce que partiellement, les relations hiérarchiques entre les thèmes.

L'analyse thématique (en tant qu'outil d'analyse linguistique) permet également de modéliser les relations entre les différents paragraphes. Dans cette perspective, la notion d'hyperthème devient centrale : elle est le dénominateur commun qui permet de regrouper dans un ensemble textuel les différents paragraphes qui, eux, représentent autant d'embranchements sous-thématiques de l'hyperthème supérieur.

Enfin, nous avons observé que le paragraphe participe aux relations compositionnelles du texte. Les éléments constitutifs de la série énumérative que nous avons analysée étaient corrélés aux segments typographiques. La disposition graphique du texte en reflète la structure compositionnelle («rhétorique») qui est ainsi signalée et donc communiquée au récepteur du texte.

Si le paragraphe est incontestablement («évidentiellement») une unité graphique, il l'est aussi pour des raisons de contenu et de composition : reste à savoir (car il est bien difficile en l'état actuel des recherches de savoir) si celles-ci précèdent donc «prédisent» celle-là.

\section{Références bibliographiques}

ADAM, J.-M. 1990. Éléments de linguistique textuelle: théorie et pratique de l'analyse textuelle. Liège : Mardaga.

Adam, J.-M. 2011 [1999]. La linguistique textuelle. Introduction à l'analyse textuelle des discours. Paris: A. Colin [3 éd.].

AdAm, J.-M. 2012. Le modèle émergentiste en linguistique textuelle. L'information grammaticale 134 : 30-37.

Adam, J.-M. 2017 [1992]. Les textes: types et prototypes. Paris: A. Colin [4éd.].

AdAM, J.-M. 2018. Le paragraphe: entre phrases et texte. Paris: A. Colin.

Algee-Hewitt, M., Heuser, R. et Moretti, F. 2015. On Paragraphs. Scale, Themes, and Narrative Form. Pampblets of the Stanford Literary Lab 10: 1-22. En ligne à l'adresse suivante: https://litlab.stanford.edu/LiteraryLabPamphlet10.pdf.

ARABYAN, M. 1994. Le paragraphe narratif: étude typographique et linguistique de la ponctuation textuelle dans les récits classiques et modernes. Paris: L'Harmattan.

Arabyan, M. (éd.) 2003. Modèles linguistiques: Le paragraphe 48. Université de Toulon.

Arabyan, M. 2012. Des lettres de l'alphabet à l'image du texte. Recherches sur l'énonciation écrite. Limoges: Lambert-Lucas.

Arabyan, M. (éd.) 2016. Semen: L'énonciation éditoriale 41. Besançon: Presses universitaires de Franche-Comté.

Benveniste, É. 1966. Problèmes de linguistique générale I. Paris: Gallimard.

Bessonnat, D. 1988. Le découpage en paragraphes et ses fonctions. Pratiques 57: 81-105. 
ČERVENKA, M. 1983. Výpovědní témata bez opory v předcházejícím textu (na materiálu české umělecké prózy). In T. Dobrzyńska et E. Janus (éd.), Tekst i zdanie. Wrocław: Ossolineum : 81-96.

Combettes, B. 1998. Les constructions détachées en français. Gap - Paris: Ophrys.

Combettes, B. 2015. Éléments pour une linguistique textuelle diachronique. In A. Ferrari, L. Lala et R. Stojmenova (éd.), Testualità. Fondamenti, unità, relazioni. Florence: Franco Cesati Editore: 249-261.

Culioli, A. 1984. En guise d'introduction. In A. Grésillon et J.-L. Lebrave (éd.), La langue au ras du texte. Lille: Presses universitaires de Lille: 9-12.

Daneš, F. 1974. Functional Sentence Perspective and the Organization of the Text. In F. Daneš (éd.), Papers on Functional Sentence Perspective. Prague - La Haye : Academia De Gruyter: 106-128.

DANEš, F. 1989. Report of Roger G. van de Velde’s Paper “Man, Verbal Text, Inferencing and Coherence”. In W. Heydrich, F. Neubauer, J. S. Petöfi et E. Sözer (éd.), Connexity and Coberence: Analysis of Text and Discourse. Berlin - New York: De Gruyter : 228-239.

DANEš, F. 1994. Odstavec jako centrální jednotka tematicko-kompoziční výstavby textu (na materiále textů výkladových). Slovo a slovesnost 55 : 1-17.

Delisle, L. 1906. Maître Jean d'Antioche, traducteur, et Frère Guillaume de Saint-Etienne, hospitalier. In Congrégation de SAint-Maur et ACAdÉmie des insCriptions et Belles-lettres (éd.), Histoire littéraire de la France. Paris: Imprimerie nationale. T. 33 : Suite du quatorzième siècle: 1-40.

Eco, U. 1985 [trad. de 1979]. Lector in fabula. M. Bouzaher (trad. de l'italien). Paris: B. Grasset.

Englebert, A. 2000. Si, car, que, et, or... signes de ponctuation de l'ancien français. In A. Englebert, M. Pierrard, L. Rosier et D. Van Raemdonck (éd.), Actes $d u$ XXII congrès international de Linguistique et de Philologie romanes, Bruxelles, 23 au 29 juillet 1998. Tübingen: M. Niemeyer. T. 2: Les nouvelles ambitions de la linguistique diachronique: $175-184$.

Enkvist, N. E. 1974. “Theme Dynamics” and Style: An Experiment. Studia Anglica Posnaniensia 5 : 127-135.

Firbas, J. 1964. On Defining the Theme in Functional Sentence Analysis. Travaux linguistiques de Prague 1: 267-280.

Firbas, J. 1992. Functional Sentence Perspective in Written and Spoken Communication. Cambridge - New York: Cambridge University Press.

Gardes-Tamine, J. et Pellizza, M.-A. 1998. De l'unité textuelle au paragraphe et au texte. In J. Gardes-TAmine et M.-A. PellizzA, La construction du texte: de la grammaire au style. Paris: A. Colin : 70-88.

Greimas, A. J. 1976. Maupassant. La sémiotique du texte: exercices pratiques. Paris: Seuil.

Groupe de Fribourg 2012. Grammaire de la période. C. Blanche-Benveniste (postface). Berne: P. Lang.

HARris, Z. S. 1952. Discourse Analysis. Language 28 (1): 1-30.

JACKIEwICZ, A. 2005. Les séries linéaires dans le discours. Langue française 148: 95-110. 
JaCQUes, M.-P. 2005. Structure matérielle et contenu sémantique du texte écrit. Corela 3 (2): 1-27. En ligne à l'adresse suivante: http://journals.openedition.org/corela/560.

LAufer, R. 1985. L'alinéa typographique du XVI e au XVIII ${ }^{e}$ siècle. In R. LAufer (éd.), La notion de paragraphe. Paris: Éditions du CNRS : 53-63.

Lavrentiev, A. 2016. Ponctuation française du Moyen Âge au XVI ${ }^{\mathrm{e}}$ siècle: théories et pratiques. In S. PÉtillon, F. Rinck et A. Gautier (éd.), La ponctuation à l'aube du XXI siècle. Perspectives historiques et usages contemporains. Limoges: Lambert-Lucas: 39-62.

Legallois, D. 2006a. Présentation générale. Le texte et le problème de son et ses unités: propositions pour une déclinaison. Langages $163: 3-9$.

Legallois, D. 2006b. Des phrases entre elles à l'unité réticulaire du texte. Langages 163: 56-70.

LindeberG, A.-C. 1991. Discourse Characteristics in EFL Composition in Upper Secondary Schools in Finland. In L. S. Evensen (éd.), Nordwrite Reports IV. Trondheim : University of Trondheim, Department of Applied Linguistics.

Llamas Pombo, E. 2001. La ponctuation du vers dans un manuscrit du XIV siècle. Liaisons HÉSO/AIROÉ 32-33: 151-171.

Longacre, R. E. 1979. The Paragraph as a Grammatical Unit. In J. P. Kimball et T. Givón (éd.), Syntax and Semantics. New York: Academic Press. Vol. 12: Discourse and Syntax: 116-134.

Luc, C. 2001. Une typologie des énumérations basée sur les structures rhétoriques et architecturales du texte. In TALN - RECITAL 2001: 8 conférence annuelle sur le Traitement automatique des langues naturelles (2-5 juillet 2001, Tours). Paris: Association pour le Traitement automatique des langues (ATALA) : 263-272. En ligne à l'adresse suivante : https://www.atala.org/sites/default/files/actes_taln/AC_0045.pdf.

Luc, C. et Virbel, J.-L. 2001. Le modèle d'architecture textuelle: fondements et expérimentation. Verbum 23 (1): 103-123.

Mathesius, V. 1969. Les thèses de 1929. Change $3:$ 21-49.

Miller, G. A. 1956. The Magical Number Seven, Plus or Minus Two. Some Limits on Our Capacity for Processing Information. Psychological Review 63 (2): 81-97.

Mitterand, H. 1985. Le paragraphe est-il une unité linguistique? In R. Laufer (éd.), La notion de paragraphe. Paris: Éditions du CNRS : 85-95.

Péry-Woodley, M.-P., Afantenos, S. D., Ho-Dac, L.-M. et Asher, N. 2011. La ressource ANNODIS, un corpus enrichi d'annotations discursives. TAL - Traitement automatique des langues 52 (3): 71-101. En ligne à l'adresse suivante: https://www.atala.org/sites/ default/files/Pery-Woodley-TAL52-3.pdf.

PEŠEK, O. 2010. La linguistique textuelle tchèque au seuil du XXI ${ }^{\mathrm{e}}$ siècle: la genèse d'une discipline et la tradition pragoise. Verbum 32 (2): 263-282.

PešEK, O. 2017. La "Rectorique de Cyceron" traduite par Jean d'Antioche: organisation du texte et son marquage. Études de linguistique textuelle diachronique. České Budějovice: Jihočeská univerzita, edice Epistémé.

Schank, R. C. 1974. Understanding Paragrapbs. Technical Report 6. Lugano: Istituto per gli studi semantici e cognitivi. En ligne à l'adresse suivante: https://www.issco.unige. ch/working-papers/Schank-1974.pdf. 
VAN Dijk, T. 1973. Text Grammar and Text Logic. In J. S. PetöFi et H. Rieser (éd.), Studies in Text Grammar. Dordrecht - Boston: D. Reidel: 17-78.

\section{Éditions}

CicÉron, De l'invention. G. Achard (éd. et trad.) 1994. Paris: Les Belles Lettres [éd. bilingue fr.-lat.].

[Cicéron, De l'invention. JeAn D’Antioche (trad. anc. fr.)] La "Rectorique de Cyceron" tradotta da Jean d'Antioche. E. GuAdAGNINI (éd.) 2009. Pise: Scuola normale superiore.

CicÉron, De l'invention. Jean d’Antioche (trad. anc. fr.). W. VAN Hoecke (éd.) 2011. Publié en ligne par la Base de français médiéval. Accessible en ligne à l'adresse suivante: http://catalog.bfm-corpus.org/JAntInv (dernière révision le 20/02/2013). 